\title{
Email in Medical Practice: A Critical Review
}

\author{
Alysha Nensi and Natasha Chandok
}

\begin{abstract}
Email between patients and physicians can be an effective means of communication and health care delivery, but concerns over security, privacy, feasibility, and legality prevail. This paper reviews the limited literature in this area, highlighting our current understanding of the prevalence of email in clinical practice, patient attitudes toward email communication, and the impact of email communication on patient outcomes. While there is a paucity of data on the role, benefits, and risks of email communication, patients and physicians alike should consider secure email as a convenient tool for communication. Physicians require further guidelines on appropriate uses of email in clinical practice to best enhance patient autonomy, preserve patient confidentiality, and comply with current medico-legal standards.
\end{abstract}

Key-words: Physician Patient Relationship, Communication, Electronic Mail, Ethics, Medical, Delivery of Health Care

\section{INTRODUCTION}

The patient-physician relationship is fundamental to the practice of medicine and medical ethics. A physician has an ethical obligation to deliver health care in a manner in which the dignity and privacy of their patients is upheld. While modern medicine rests on the four pillars of nonmaleficence, beneficence, autonomy, and justice, our society places increasing value on patient autonomy. Communication is at the heart of patient autonomy; it allows for the exchange of information and empowers patients to be active participants in their own care. Communication between both patient and physician is enhanced when both parties share mutual respect, knowledge, trust, and values.

Anumber of issues, however, complicate the patient-physician relationship. In busy ambulatory clinics, for example, health care providers may not have the ability to spend a sufficient amount of

To whom correspondence should be addressed:

Natasha Chandok, MD, MPH

Assistant Professor of Medicine

Division of Gastroenterology

University of Western Ontario

339 Windermere Road, London, Ontario

N6A5A5 Canada

natasha.chandok@Ihsc.on.ca time with each patient. Physicians may need to see too many patients in a given time frame, and are typically burdened with extensive paper work and other documentation that takes time away from face-to-face patient-physician encounters. Different settings of health care may add further complexity to the patient-physician relationship. For instance, in the hospital ward or intensive care unit setting, a number of other individuals may be involved in the care of a patient, including relatives, friends, nurses, social workers and other specialists. With multiple participants, it may be difficult for patients and their families to assimilate information and identify the most responsible physician. Another barrier to communication is classism; patient and/or physicians may perceive physicians as superior on the basis of education, expertise and socioeconomic status. Likewise patients may be perceived as inferior to their doctors on the basis of their physical or emotional suffering, and potential lack of knowledge and lower socioeconomic status. These types of barriers make it difficult for patients to ask questions and receive the information they need to be active participants in their own care.

The communication between patients and physicians faces new challenges in this information era, where patients are more educated and involved 
with their health care and have access to a wealth of medical knowledge at their fingertips because of the internet. With email and other communication interfaces on the internet, patients can also reach self-help and support groups and medical experts from around the world. Despite major advancements in communications, many patients and physicians do not communicate with one another via email, and medical laws have not kept pace with changes in communication technologies for the delivery of health care.

Email offers a number of potential advantages to enhancing the patient-physician relationship. For example, patients and physicians can connect more efficiently with one another, unlike telephone encounters where both parties need to be available at the same time. Email also offers the opportunity for patients to receive more education and general advice, which may be restricted in a face-to-face or telephone exchange where obstacles such as shortages of doctors, and time restraints on clinic appointments, are all too common. As such, email communication may improve patient satisfaction, compliance with therapy, minimize preventable errors, and could potentially be cost-effective. Email may also foster a patient's personal involvement in their own care and encourage self-care. To date, the impact of email on the therapeutic relationship between the physician and patient has not been thoroughly studied.

Physicians and patients need guidelines on the appropriate role of email in health care delivery. Email is likely a suitable forum through which a patient can make an appointment, ask a question or clarify an instruction, receive education, receive reminder for appointment, and enable a health care provider to monitor progress while undergoing treatment. However, it is important to note that email should not serve in a capacity which jeopardizes the delicate balance of the patientphysician relationship. As such, email should not substitute a face-to-face encounter where complicated or abnormal tests are reviewed, or a diagnosis, prognosis, or treatment information is shared with a patient.

Issues of security and confidentiality are primary concerns of patients and physicians who communicate with one other via email. Exposure of confidential health information to a patient's employer, another family member, marketer, or insurance company can have personal ramifications for a patient, and result in litigation against a physician. Ideally, patients should have a dedicated email address for the exchange of medical information with their doctor, and data containing a patient's health information should be encrypted. For example, an email interface could help triage messages through the use of a drop down menu to indicate the level of urgency of the message. An email interface can automatically instruct a patient to call their doctor's office rather than submit an email for an urgent health care matter, and interfaces can limit the number of words included in a health care message so that physicians do not need to spend an elaborate amount of time on each email. Thus, in light of these issues, individual health care institutions and medical societies may need to consider developing training protocols and general policies to protect a patient's privacy.

There are a number of potential pitfalls to email communication between patients and their doctors as well. For example, it is unknown whether email may lead to an increase health care utilization, and how time intensive email communications will be for health care providers in busy practices. There are also possible medicolegal ramifications if physicians do not respond in a timely manner, or if advice dispensed over email is unclear for patients. Another important concern is that email communication could lead to health care disparities between patients of higher versus lower socioeconomic status, or patients who do not speak English fluently and, therefore, may not be able to compose an email their physician.

For a myriad of reasons, some patients and physicians are reluctant to embrace email as a means of communication. That being said, email may potentially offer an opportunity to improve patient satisfaction with health care and enhance communication in the patient-physician relationship. The purpose of this paper is to review the current understanding of the impact of email between patients and physicians for the delivery of health care.

\section{METHODOLOGY}

Using MEDLINE with no date limits, a literature search was conducted using the medical subject headings: "electronic mail", "physician patient relationship", "ethics", "communications", and "delivery of health care". Studies were screened upon review of their title and abstract. After a review of the article, studies were included if they met the following inclusion criteria: the article was written in English; the patient population was 
greater than or equal to 18 years of age; outcomes included patient use, satisfaction or interest with electronic mail as a means for communication; or outcomes included the impact of electronic mail on medical management. Editorials and clinical review articles generated from the search were excluded but nevertheless reviewed for their content and reference lists, which were examined to identify any original articles which fulfilled inclusion criteria not found on the original search.

\section{RESULTS}

The search yielded 10 studies that fulfilled the inclusion criteria. Three studies examined the prevalence or patient attitudes of email usage in clinical practice. In a cross-sectional cohort study of all adult patients at an integrated health-care delivery system, Ralston et al. found that only $14 \%$ of 25,075 patients exchanged one or more e-mails with their primary or specialty care provider over a 14 month period (1). Factors associated with an increased likelihood of exchanging secure email included female gender, greater overall morbidity, and the health care provider's use of email with other patients (1). Less secure email use was associated with patient age over 65, and Medicaid insurance rather than commercial insurance (1). In a second cross-sectional study in 2005 from a large multispecialty group practice, investigators found that while $58.3 \%$ of 186,000 patients had email access, only $5.8 \%$ reported ever using email to communicate with their physician(s) (2). Patients were most willing to use email to obtain cholesterol and blood sugar tests, but were less keen on receiving emails containing medical imaging (e.g. computed tomography scan) test results (2). A third study from 1994, albeit now outdated, found that $46 \%$ of patients in an internal medicine practice regularly used email, and $89 \%$ reported email use only at work (3). $51 \%$ reported that they would use email all the time or most of the time if it was available as a means of communication with their health care providers (3).

Three studies examined patient use of email, and attitudes toward incorporating email into their health care delivery strategy. In a 2008 study in an outpatient rheumatology clinic, $74.5 \%$ of patients reported that they had internet access, and $72 \%$ of the 127 patients stated they would want to communicate with their rheumatologist with email, while only $28 \%$ reported a complete lack of interest in email communication (4). $41 \%$ of respondents identified privacy as a major concern with email (4). Younger adults and patients whose insurance would cover email communications (as opposed to paying out of pocket) were more likely to desire email communication with their rheumatologist. In this study, interest in emailing their rheumatologist or having access to the internet was not associated with a patient's education, income, or gender. In a third study, a randomized controlled trial from the University of Colorado, investigators examined the impact of an internet-based patient portal on patient satisfaction with access to their clinical care versus standard-of-care telephone communications (5). Patients in the "portal" group, who had access to secure emails to their health care providers, reported improved communication (44\% versus $12 \%, p<0.001)$ and higher satisfaction with their overall health care $(59 \%$ versus $48 \%, p=0.04)$ as compared to the control group (5).

One study examined the content of emails by health care providers and health care staff, providing insight into the proportion of emails a primary care practice composes to specifically communicate with patients and their families. In a diabetes care clinic at an American academic medical center, 27,061 emails exchanged over a 6 month period in 2003 between health care providers and staff caring for a cohort of 639 patients were analyzed to determine their content (6). Stiles et al. found that $47.2 \%$ of emails were done to communicate with patients, families, and other providers; the remainder of the emails were done between clinic staff for a variety of reasons such as documentation, logistics, and support functions (6).

One study investigated the use of unsolicited email from patients or their families who were seeking medical advice from expert physicians who published their research on the internet, on the only internet site in 1995 with a primary focus on cardiac arrhythmias (3). This study documented 70 unsolicited emails from 39 patients and 20 family members over a 12 month period; 22 inquiries received specific follow up advice (3). This study demonstrates that some patients and their families are capable of and interested in seeking subspecialty medical advise over email, and that physicians require guidelines on the appropriateness of dispensing recommendations over email (3).

Two studies investigated the role of email in enhancing patient care outcomes. In a trial of 83 adults with type 2 diabetes mellitus, Ralston et al. randomized patients to receive usual care plus web-based care versus usual care alone (7). In this study, the web-based care included patient access to medical records, secure email with providers, 
email feedback on blood glucose readings, and an interactive online diary to enter health care information such as diet and exercise (7). Patients who received access to secure web communications had a statistically significant improvement in their glycemic control (hemoglobin A $1 \mathrm{C}$ decline of $0.7 \%$, $95 \% \mathrm{Cl} 0.2-1.3)$ as compared to patients who received standard of care only (7). In a second study focusing on email in an addiction medicine clinic, Collins et al. found that email communications between patients and their specialist can enhance treatment for substance dependency through a variety of means (8). For example, with the free ability to email their providers, patients can share daily self-assessments and receive continual encouragement and advice to amplify their odds of maintaining sobriety (8).

\section{DISCUSSION AND CONCLUSION}

The technology exists for secure, efficient electronic communications between patients and their health care providers through a variety of means, including email. While issues of concern over patient privacy must be discussed between the patient and health care provider, it is clear that many patients are interested in incorporating email communication into their health care experience. Email holds the potential to significantly improve health care delivery by facilitating communication between patients and their providers, thus enhancing the patient-physician relationship and improving patient satisfaction. Furthermore, email communications can also lead to improved clinical outcomes for patients.

Unfortunately, there is a paucity of data examining the role of email, and the impact of email on health care and patient outcomes. The assessment of the literature on this topic conducted for this review generates a number of questions, including the role of patient gender, socioeconomic status, and computer literacy on email preference for health related communications with practitioners. Further studies are needed to assess these important questions, as well as the cost-effectiveness and broader role of email in clinical practice.

Additionally, data is needed to guide expert panels in the development of guidelines for practitioners on the appropriateness and use of email in clinical practice. It is particularly imperative that the adoption of email in standard communications between physicians and patients not impinge upon the patient-physician dynamic or generate health disparities among patients of lower socioeconomic status. Despite the many challenges in establishing email as a widespread standard adjuvant practice in health care delivery, email has the potential to aid in health promotion and disease prevention.

\section{CONFLICTS OF INTEREST}

The authors declare that they have no conflicts of interest.

\section{REFERENCES}

1. Ralston JD, Rutter CM, Carrell D, Hecht J, Rubanowice $D$, Simon GE. Patient use of secure electronic messaging within a shared medical record: a cross-sectional study. J Gen Intern Med. 2009 Mar;24(3):349-55.

2. Couchman GR, Forjuoh SN, Rascoe TG, Reis MD, Koehler B, Walsum KL. E-mail communications in primary care: what are patients' expectations for specific test results? Int J Med Inform. 2005 Jan;74(1):21-30.

3. Widman LE, Tong DA. Requests for medical advice from patients and families to health care providers who publish on the World Wide Web. Arch Intern Med. 1997 Jan 27;157(2):209-12.

4. Siva C, Smarr KL, Hanson KD, Parikh M, Lawlor K, Ge B. Internet use and e-mail communications between patients and providers: a survey of rheumatology outpatients. J Clin Rheumatol. 2008 Dec;14(6):318-23.

5. Lin CT, Wittevrongel L, Moore L, Beaty BL, Ross SE. An Internet-based patient-provider communication system: randomized controlled trial. J Med Internet Res. 2005;7(4):e47.

6. Stiles RA, Deppen SA, Figaro MK, Gregg WM, Jirjis JN, Rothman RL, et al. Behind-the-scenes of patient-centered care: content analysis of electronic messaging among primary care clinic providers and staff. Med Care. 2007 Dec;45(12):1205-9.

7. Ralston JD, Hirsch IB, Hoath J, Mullen M, Cheadle A, Goldberg HI. Web-based collaborative care for type 2 diabetes: a pilot randomized trial. Diabetes Care. 2009 Feb;32(2):234-9.

8. Collins GB, McAllister MS, Ford DB. Patient-provider e-mail communication as an adjunctive tool in addiction medicine. J Addict Dis. 2007;26(2):45-52. 